i.e. $S_{n}^{r}=\frac{n^{r+1}}{r+1}+\frac{1}{2} n^{r}+\sum_{k=1}^{r-1}(-1)^{k+1} \frac{r !}{(r-k) !} n^{r-k}\left|\begin{array}{ccccc}\frac{1}{2 !} & 1 & 0 & \ldots & 0 \\ \frac{1}{3 !} & \frac{1}{2 !} & 1 & \ldots & 0 \\ \ldots & \ldots & \ldots & \ldots & \ldots \\ \ldots & \ldots & \ldots & \ldots & 1 \\ 2 ! & 1 \\ \frac{1}{(k+2) !} & \ldots & & \frac{1}{3 !} & \frac{1}{2 !}\end{array}\right|$

This is the desired result, although in some respects the equivalent result (2) is preferable to (3), for apart from the final elements, the successive rows (except the last) are merely those of the well-known Arithmetical Triangle without the final 1 in each case.

Arakan,

134 Halbeath Road, DUNFERMLine.

\title{
Inequalities for solutions of linear differential equations A contribution to the theory of servomechanisms
}

By HaNs B̈̈̈rNer.

§1. Introduction.

Consider the $n^{\text {th }}$ order differential equation

$$
y+c_{1} y^{\prime}+c_{2} y^{\prime \prime}+\ldots+c_{n} y^{(n)}=f(x),
$$

where the coefficients $c_{v}$ are real constants and $f$ is a real function continuous in the interval $a \leqq x \leqq b$. The following theorem will be proved in $\$ 4$ :

If the characteristic equation of (1) has no purely imaginary roots, then a particular integral $\eta(x)$ can always be found which satisfies the inequality

$$
|\eta| \leqq C M,
$$

where $C$ is a certain function of the $c_{\nu}$ only and $M$ is the maximum of $|f|$. In particular we may take $C=1$ if all roots of the characteristic equation are real. 
It is, of course, abvious that there is a number $C$, depending only on the $c_{y}$, for which (2) is true; the point of the theorem is that an expression for $C$ is given (see $\$ 4$ ).

The result has an application to the theory of servomechanisms.

\$2. The 1 st order differential equation: ${ }^{1}$

$$
y+c_{1} y^{\prime}=f(x) \text {. }
$$

(a) If $c_{1}>0$, a suitable particular integral is

$$
\eta(x)=\int_{a}^{x} \frac{f(s)}{c_{1}} e^{-\frac{x-s}{c_{1}}} d s,
$$

for which it is easily verified that $|\eta| \leqq M$ and further that

$$
\eta(a)=0 \text {. }
$$

(B) If $c_{1}<0$, we replace $a$ by $b$ in the definition of $\eta(x)$. Then $\eta \eta \leqq$ and

$$
\eta(b)=0 .
$$

§3. The 2 nd order differential equation:

$$
y+c_{1} y^{\prime}+c_{2} y^{\prime \prime}=f(x) .
$$

Suppose that the characteristic equation $1+c_{1} w+c_{2} w^{2}=0$ has conjugate complex roots $\sigma \pm i \tau, \sigma \neq 0, \tau>0$.

Then $c_{2}=\left(\sigma^{2}+\tau^{2}\right)^{-1}>0$.

(a) Let $\sigma<0$. A solution of (4) satisfying the conditions

$$
\eta(a)=\eta^{\prime}(a)=0
$$

is

$$
\eta(x)=\frac{1}{c_{2} \tau} \int_{0}^{x-a} e^{\sigma \xi} \sin \tau \xi f(x-\xi) d \xi .
$$

Since $|\eta| \leqq \frac{M}{c_{2} \tau} \int_{0}^{\infty} e^{\sigma \xi}|\sin \tau \xi| d \xi=\frac{M}{c_{2} \tau} \sum_{k=0}^{\infty} e^{\frac{k \pi \sigma}{\tau}} \int_{0}^{\pi / \tau} e^{\sigma \xi} \sin \tau \xi d \xi$, it follows that (2) is satisfied with

$$
C=\operatorname{coth} \frac{1}{2} \pi|\sigma| / \tau .
$$

( $\beta$ ) Let $\sigma>0$. We replace $a$ by $b$ in the definition of $\eta$ and thus obtain a solution of (4) satisfying (2) with the value of $C$ given by (5) and satisfying also

$$
\eta(b)=\eta^{\prime}(b)=0 .
$$

1 The author has previonsly shown that the result is true, more generally, if $c_{1}$ is a continuous non-vanishing function of $x$ : see Zeitschrift für angewandte Mathematil: und Mechanili, 22 (1942), 143-152 (Hilfssat:, 144). 
\$4. The $n^{\text {th }}$ order differential equation:

$$
y+c_{1} y^{\prime}+\ldots+c_{n} y^{(n)}=f(x) .
$$

Denote the roots of the characteristic equation $1+c_{1} w+\ldots+c_{n} w^{n}=0$ by $w_{1}, \ldots, w_{n}$, and suppose ${ }^{1}$ that the first $2 m$ roots are the conjugate complex numbers

$$
w_{2 k-1}=\sigma_{k}+i \tau_{k}, \quad w_{2 k}=\sigma_{k}-i \tau_{k} \quad(k=1, \ldots, m),
$$

and that the remaining roots are real. By hypothesis

$$
\sigma_{k} \neq 0 \quad(k=1, \ldots, m) .
$$

Consider the following system of differential equations:

$$
\left.\begin{array}{rl}
\left(D-w_{2 k-1}\right)\left(D-w_{2 k}\right) Z_{k} & =Z_{k+1} \quad(k=1, \ldots, m), \\
\left(D-w_{m+k}\right) Z_{k} & =Z_{k+1} \quad(k=m+1, \ldots, n-m-1), \\
\left(D-w_{n}\right) Z_{n-m} & =f(x) / c_{n} .
\end{array}\right\}
$$

Obviously $Z_{1}$ satisfies (1). We think now of a solution $Z_{1}, \ldots, Z_{n-m}$ of (6) determined in such a way that

$$
\begin{aligned}
& \left|Z_{m+k}\right| \leqq\left|w_{2 m+k}\right|^{-1} \operatorname{Max}\left|Z_{m+k+1}\right| \quad(k=1, \ldots, n-2 m-1), \\
& \left|Z_{n-m}\right| \leqq\left|w_{n}\right|^{-1} \operatorname{Max}\left|f(x) / c_{n}\right|, \\
& \left|Z_{k}\right| \leqq \operatorname{coth} \frac{1}{2} \pi\left|\sigma_{k} / \tau_{k}\right| . \operatorname{Max}\left|Z_{\dot{k}+1}\right| \quad(k=1, \ldots, m) .
\end{aligned}
$$

There is such a solution, in riew of the results of $\$ \S 2,3$. From these inequalities, $\left|Z_{1}\right| \leqq C M$ where

$$
C=\prod_{k=1}^{m} \operatorname{coth} \frac{1}{2} \pi\left|\sigma_{k} / \tau_{k}\right| \text {. }
$$

The result stated in $\S l$ is thus proved.

§5. The $n^{\text {th }}$ order differential equation (continued).

We now consider the case when every root of the characteristic equation is negative or has a negative real part. The integral $\eta=Z_{1}$ which satisfies the inequality $|\eta| \leqq C M$, where $C$ is given by (7), then belongs to the solution $Z_{1}, \ldots, Z_{n-m}$ of the equations (6) satisfying

$$
\begin{array}{ll}
Z_{k}(a)=Z^{\prime}{ }_{k}(a)=0 & (k=1, \ldots, m), \\
Z_{m+k}(a)=0 & (k=1, \ldots, n-2 m) .
\end{array}
$$

This follows easily from the conditions (3.1) and (4.1). We thus obtain the first part of the following theorem, the second part of which can be proved similarly:

1 Only trivial modifications are required if the roots are all real or all umreal. 
The particular integral $\eta(x)$ of the theorem of $\$ 1$ can be chosen in such a way that

$$
\eta(a)=\eta^{\prime}(a)=\ldots=\eta^{(n-1)}(a)=0,
$$

if every root of the characteristic equation is negative or has a negative real part; or that

$$
\eta(b)=\eta^{\prime}(b)=\ldots=\eta^{(n-1)}(b)=\mathbf{0},
$$

if every root is positive or has a positive real part.

\section{§. Application.}

Suppose that $f(x)$ is continuous and $|f(x)| \leqq M$ in $0 \leqq x<\infty$, and that every root of the characteristic equation of (1) is negative or has a negative real part. Then, if $y(x)$ is any particular integral of (1),

$$
\varlimsup_{x \rightarrow \infty}|y(x)| \leqq C M,
$$

where $C$ is given by (7).

By what has been already proved, a particular integral $\eta(x)$ can be found such that $|\eta(x)| \leqq C M$. Moreover, we may write $y(x)=\eta(x)+y_{0}(x)$, where $y_{0}(x)$ is the complementary function. The required result then follows, for, since the real parts of the roots of the characteristic equation are negative, $y_{0}(x) \rightarrow 0$ as $x \rightarrow \infty$.

This theorem can be applied to many devices and to all stable servomechanisms that satisfy a linear differential equation with constant coefficients. In such servomechanisms $y$ represents the reponse to the disturbance $f(x)$, and the theorem shows that ultimately the absolute value of the response does not sensibly exceed $C M$. This gives a useful inequality for $y$.

Finally, we observe that, for complex roots for which $\left|\tau_{k}\right| \leqq\left|\sigma_{k}\right|$, $\operatorname{coth} \frac{1}{2} \pi\left|\sigma_{k} / \tau_{k}\right|$ does not differ essentially from unity.

\section{GöTtingen.}

